Meta

Journal des traducteurs

Translators' Journal

\title{
Terminologie comparée anglais-français de la vision par ordinateur et des sujets connexes (deuxième partie)
}

Jacques Lethuillier et Monique C. Cormier

Volume 38, numéro 4, décembre 1993

Le Je du traducteur

The $I$ of the Translator

URI : https://id.erudit.org/iderudit/003495ar

DOI : https://doi.org/10.7202/003495ar

Aller au sommaire du numéro

Éditeur(s)

Les Presses de l'Université de Montréal

ISSN

0026-0452 (imprimé)

Découvrir la revue

Citer ce document

Lethuillier, J. \& Cormier, M. C. (1993). Terminologie comparée anglais-français de la vision par ordinateur et des sujets connexes (deuxième partie). Meta, 38(4), 677-704. https://doi.org/10.7202/003495ar d'utilisation que vous pouvez consulter en ligne. 


\section{ÉTUDES TERMINOLOGIQUES ET LINGUISTIQUES}

\section{TERMINOLOGIE COMPARÉE ANGLAIS-FRANÇAIS DE LA VISION PAR ORDINATEUR ET DES SUJETS CONNEXES (deuxième partie)}

machine learning

machine vision, computational vision, computer vision

magnification - Syn. de dilation.

magnification ratio

magnifying power

Mahalanobis distance

Manhattan metric

mapping

mark

mask - Syn. de template.

masking

MAT - Syn. de skeletonization.

mat surface - Syn. de diffuse surface.

matched filter

matched filtering templating, template matching, template filtering

matching template - Syn. de template.

mathematical morphology

mathematical operator

matrix camera

matrix determinant

matrix transpose

matte surface - Syn. de diffuse surface.

maximal clique

mean square distance

mean vector

class mean vector

Meta, XXXVIII, 4, 1993 apprentissage automatique, ASA, apprentissage symbolique automatique

visionique, VO, vision artificielle, vision par ordinateur

grandissement du capteur optique

grossissement

distance de Mahalanobis

distance de Manhattan, distance quadrillée, distance Manhattan

application

marque

masquage

filtre adapté

filtrage adapté, accord de modèles

morphologie mathématique

opérateur algébrique

caméra matricielle,

caméra à état solide à cible matricielle

déterminant d'une matrice

transposée de matrice

clique maximale

distance moyenne des carrés

vecteur-moyenne, vecteur moyen 
measurement vector - Syn. de pattern vector.

medial axis transform - Syn. de skeletonization.

medial axis transformation - Syn. de skeletonization.

$\begin{array}{ll}\text { median filter } & \text { filtre de la médiane, filtre médian } \\ \text { medical imagery } & \text { imagerie médicale } \\ \text { membership } & \text { appartenance } \\ \text { merge-only quad-tree segmentation }- \text { Syn. de region merging. } \\ \text { metric distance, } & \text { métrique, fonction distance, } \\ \text { distance, distance function } & \\ \text { metric space } & \text { espace métrique } \\ \text { metrizable topological space } & \text { espace topologique métrisable } \\ \text { microdensitometer } & \text { microdensitomètre } \\ \text { microphotography } & \text { microphotographie } \\ \text { microscopy } & \text { microscopie } \\ \text { microtome } & \text { microtome } \\ \text { minimax criterion } & \text { critère du minimax } \\ \text { minimum-distance classifier, } & \text { classifieur par distance minimale }\end{array}$

distance classifier

Minkadd operation - Syn. de Minkowski addition.

Minkowski subtraction - Syn. de erosion.

mirror reflexion - Syn. de specular reflection.

mode-locked laser

model

modulation transfer function, $M T F$

moiré

moment

morphological filter

morphological operation

motion blur

motion picture camera - Syn. de camera.

motion-picture camera - Syn. de camera.

movie-camera - Syn. de camera.

moving camera

MSS - Syn. de multispectral scanning system.

$M T F$ - Syn. de modulation transfer function. laser à modes synchronisés

modèle

fonction de transfert de, modulation réponse (d'un film), F.T.M.

moiré, effet de moiré

moment

filtre morphologique

opération morphologique

flou de bougé

caméra mobile 
Multi Spectral Scanner - Syn. de multispectral scanning system.

multi-image averaging, multiple image averaging, averaging

multiple edges moyennage d'images

multiple image averaging - Syn. de multi-image averaging.

multiplet

multiplet

multispectral image

image multispectrale

multispectral scanning system,

radiomètre multispectral à balayage

Multi Spectral Scanner, MSS

N.A. - Syn. de numerical aperture.

nanometer

nanomètre

natural light

lumière naturelle

near object, nearby object

objet rapproché

near point of vision

distance minimale de vision distincte, punctum proximum

near-infrared, near-IR

infrarouge proche

near-IR - Syn. de near-infrared.

nearest neighbor classification, classification par la méthode des $\mathrm{N}$

NN classification

plus proches voisins

nearest neighbor classification rule, décision par plus proche voisin nearest-neighbor decision rule

nearest-neighbor decision rule - Syn. de nearest neighbor classification rule.

neighborhood

voisinage

neighborhood family

famille de voisinage

neighborhood processing — Syn. de group processing.

neighborhood transformation - Syn. de group processing.

network - Syn. de directed graph.

Newton's rings anneaux de Newton

NN classification - Syn. de nearest neighbor classification.

nodal point

point nodal

node, vertex

nœud, sommet

node expansion,

développement d'un nœud

node generation

rode (of a vibration) trough

nœud de vibration

noise - Syn. de image noise.

noise-corrupted image — Syn. de noisy image.

noisy image, noise-corrupted image image bruitée 
nonterminal alphabet - Syn. de nonterminal set.

nonterminal set, nonterminal alphabet

normal distribution,

Gaussian distribution

normal probability density function, Gaussian probability, density function normal vector vocabulaire non terminal, alphabet auxiliaire, alphabet non terminal

distribution normale

fonction de densité normale, fonction de densité de probabilité normale vecteur normal

Nyquist criterion - Syn. de sampling theorem.

Nyquist frequency

object

object boundary, object edge, contour

object edge - Syn. de object boundary.

object identification

object labelling

object location

object point

object recognition

object space

objective, object glass, lens fréquence de Shannon

objet

contour d'objet

identification d'objets

étiquetage des objets

localisation d'objets

point-objet

reconnaissance d'objets

espace objet

objectif

observation vector - Syn. de pattern vector.

observer - Syn. de viewer.

occluding contour, occluding edge, contour d'occultation, occultation occlusion, occluding line

occluding edge - Syn. de occluding contour.

occluding line - Syn. de occluding contour.

occlusion - Syn. de occluding contour.

ocular (adj.)

oculaire (adj.)

off dot

point éteint

on dot

point allumé

one-to-one mapping — Syn. de injection mapping.

onto mapping - Syn. de surjective mapping.

open operation - Syn. de opening operation.

opening operation, open operation ouverture

ophtalmometry

ophtalmométrie

ophtalmoscope

ophtalmoscope 
optic axis, central projection axis, optical axis, principal axis

optical axis - Syn. de optic axis.

optical bench

optical center

optical contact

optical density

optical fiber, light guide

optical filter

optical glass

optical image

optical lever

optical microscope, photon microscope, light microscope

optical path, optical distance, optical length

optical path difference, optical retardation

optical picture processing - Syn. de optical processing.

optical processing,

optical pictureprocessing

optical rangefinder

optical rotary power, optical activity

optical sensitization

optical sensor, light detector,

light sensor

optical surface

optical system

optical twinning, chiral twinning

ordinary ray

orthogonal transformation

output image

overexposure

parallel computation

parallelism axe optique

banc d'optique

centre optique

contact optique

densité optique

fibre optique

filtre optique

verre optique

image optique

miroir tournant

microscope optique

chemin optique

différence de marche traitement optique d'image, traitement optique

télémètre optique

pouvoir rotatoire

sensibilisation, sensibilisation spectrale

capteur de lumière, capteur optique

dioptre

système optique

maclage

rayon ordinaire

transformation orthogonale

image de sortie

sur-exposition

calcul parallèle

parallélisme

parameter image - Syn. de intrinsic image. 
paraxial approximation

parsing, syntax analysis

passive stereo vision

path - Syn. de trail.

path

pattern - Syn. de pattern vector.

pattern class $w w$

pattern classification

pattern classifier,

pattern recognition network, categorizer approximation de Gauss, conditions de Gauss, approximation paraxiale

analyse syntaxique

stéréovision passive

chemin

classe de formes, famille de formes

classification

classifieur

pattern description language - Syn. de picture description language.

pattern primitive - Syn. de primitive shape.

pattern recognition

reconnaissance de formes, reconnaissance automatique des formes, reconnaissance des formes

pattern recognition network - Syn. de pattern classifier.

pattern similarity measure - Syn. de similarity measure.

pattern vector, representation pattern vector, vecteur d'attributs numériques, observation vector, feature vector, observation, vecteur d'attributs pattern, vector, measurement vector

$P D L$ - Syn. de picture description language.

perfect image - Syn. de ideal image.

perfect input image - Syn. de ideal image.

perfect radiator - Syn. de blackbody.

persistence - Syn. de lag.

persistence of vision

persistance de l'image rétinienne, rémanence des impressions visuelles, persistance des impressions visuelles, persistance rétinienne, persistance des impressions rétiniennes

perspective distorsion distorsion perspective

perspective projection projection perspective

perspective transform - Syn. de perspective transformation.

perspective transformation,

transformation perspective perspective transform

perspective transformation matrix

matrice de perspective, matrice perspective de la caméra, matrice perspective, matrice de transformation perspective 


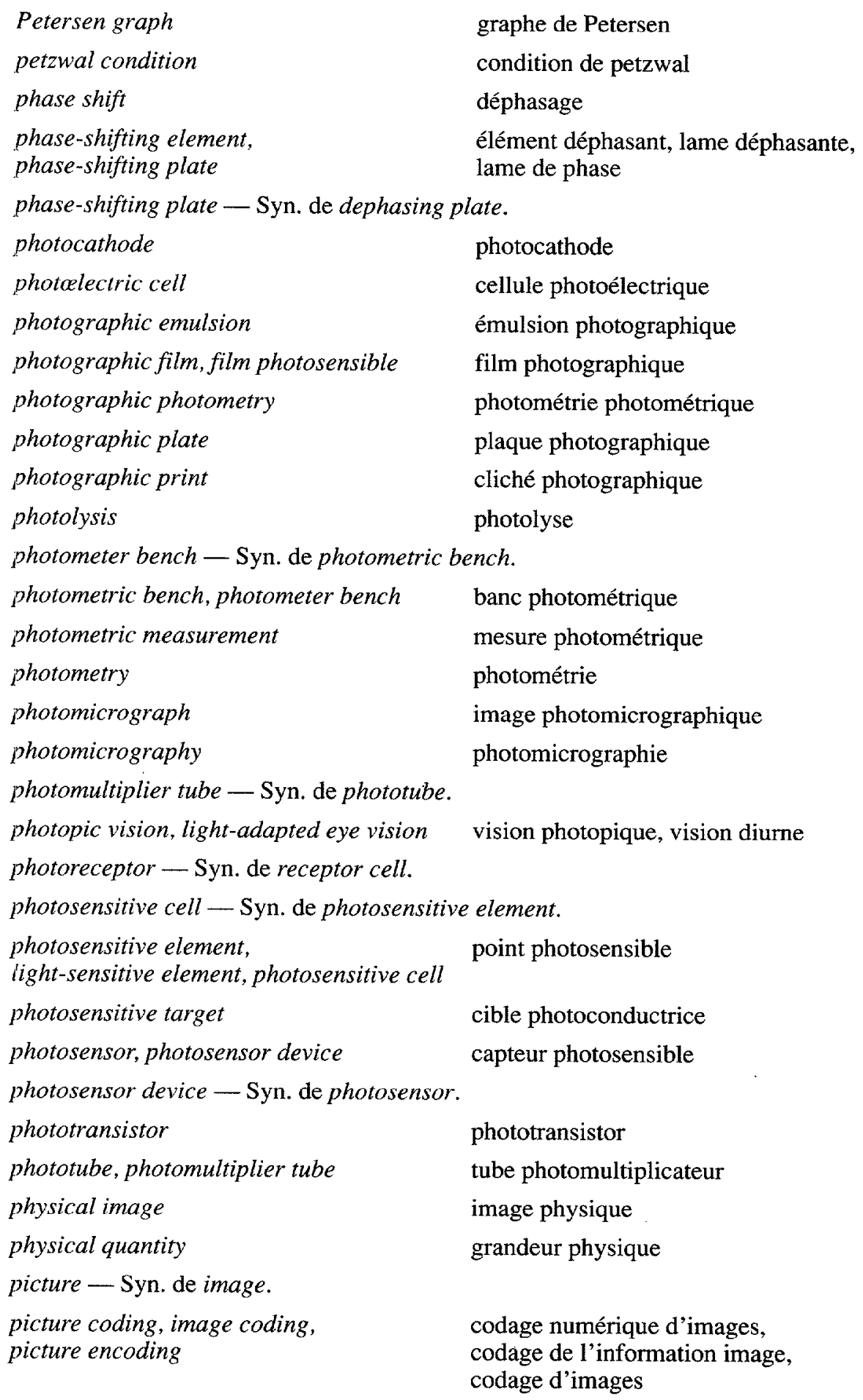


picture compression - Syn. de compression.

picture description language,

langage de description d'images

picture language,

pattern description language, $P D L$

picture element - Syn. de pixel.

picture encoding - Syn. de picture coding.

picture enhancement - Syn. de image enhancement.

picture function - Syn. de image.

picture language - Syn. de picture description language.

picture processing - Syn. de image processing.

picture taking process prise de vue, prise d'image

pin-hole model - Syn. de pinhole-camera model.

pincushion distorsion

distorsion en croissant,

distorsion en coussinet

pinhole

sténopé

pinhole-camera model, pinhole-lens model, pin-hole model modèle du sténopé, modèle à sténopé, modèle sténopé

pinhole-lens model - Syn. de pinhole-camera model.

pixel, picture element

pixel, point élémentaire,

point-image, point image

pixel group processor, group processor

convolueur

pixel transformation - Syn. de point processing.

planar graph

graphe planaire

Planck's constant

constante de Planck

plane mirror

miroir plan

plane of polarization

plane polarization, linear polarization

plan de polarisation

plane-parallel plate, parallel-sided plate

plane-polarized light,

linearly polarized light

planetary probe

polarisation totale, polarisation rectiligne

lame à faces parallèles

plumbicon

point light source

point of inflection

lumière polarisée rectilignement

point operation

point processing, pixel transformation

sonde spatiale

tube Plumbicon

source ponctuelle

point d'inflexion

opération ponctuelle

traitement ponctuel 
point of sampling, sample point

point d'échantillonnage

point spread function - Syn. de impulse response.

point-to-point distance

distance point à point

point-to-set distance

distance point à ensemble

polariscope

polariscope

polarization

polarisation

polarized light

lumière polarisée

polarizibility ellipsoid,

reciprocal ellipsoid, indicatrix,

ellipsoïde des indices

index ellipsoid

polarizing microscope

microscope polarisant

polygonal approximation,

polygonal approximation,

approximation of contours

approximation polygonale, polygonale des contours

polygonal approximation of contours - Syn. de polygonal approximation.

power of resolution, resolving power

pouvoir séparateur, limite de résolution

power spectral density - Syn. de power spectrum.

power spectrum, power spectral density,

Wiener spectrum

spectre de puissance, DSB,

densité spectrale du bruit,

densité spectrale de puissance,

spectre de Wiener

Poynting vector

vecteur de Poynting

prefiltering

préfiltrage passe-bas, préfiltrage,

préfiltrage basses fréquences,

préfiltrage induit

preprocessing, image preprocessing

prétraitement, corrections, prétraitement des images, prétraitement de l'image

preprocessor module de prétraitement

primal feature - Syn. de primitive image element.

primal sketch esquisse fondamentale

primary color couleur primaire

primitive feature - Syn. de primitive image element.

primitive image element, primitive feature, primal feature

primitive shape, terminal shape element, pattern primitive

primitive d'image

descripteur de forme, attribut de forme, élément de forme, forme caractéristique, forme primitive

principal axis - Syn. de optic axis. 


$\begin{array}{ll}\text { principal point, Gauss point } & \text { point principal } \\ \text { printer } & \text { tireuse } \\ \text { printing by contact } & \text { tirage chimique par contact } \\ \text { prior knowledge - Syn. de } \text { a priori knowledge. } \\ \text { prior probability - Syn. de a priori probability. } \\ \text { prism binocular } & \text { jumelle à prismes } \\ \text { probabilistic pattern, classification } & \text { classification probabiliste } \\ \text { probability density function } & \text { fonction de densité de probabilité } \\ \text { production, rewriting rule } & \text { règle de production } \\ \text { projection } & \text { projection } \\ \text { projection geometry, projective geometry } & \text { géométrie projective } \\ \text { projection lens, projector lens } & \text { lentille de projection }\end{array}$

projection printer - Syn. de enlarger.

projective geometry - Syn. de projection geometry.

projector lens - Syn. de projection lens.

prototype - Syn. de template.

prototype, prototype point,

prototype, reference vector

point prototype, point-image

prototype point - Syn. de prototype.

point-image de référence

PSF — Syn. de impulse response.

pulsatance - Syn. de angular frequency.

pumping lemma

lemme de l'étoile

pushdown automaton

automate à pile

quad-tree - Syn. de quadtree.

quadtree, quad-tree, quadtree

arbre quaternaire, quad-tree

quantity of illumination - Syn. de light exposure.

quantization, brightness quantization

quantification

quantization level

niveau de quantification

quarter-wave plate

lame quart d'onde

radar

radar

radial histogram,

histogramme des rayons croissants

radial intensity histogram

radial intensity histogram - Syn. de radial histogram.

radiance, radiant sterance,

luminance énergétique, radiance, scene brightness luminance énergétique spectrique directionnelle 
radiant emittance - Syn. de exitance.

radiant exitance

exitance énergétique

radiant flux, radiant power

flux énergétique

radiant incidence - Syn. de irradiance.

radiant intensity

intensité énergétique,

intensité énergétique de rayonnement

radiant power - Syn. de radiant flux.

radiant reflectance - Syn. de reflectance.

radiant sterance - Syn. de radiance.

radiator

radiateur

radiographic image

image radiologique médicale

radiometer

radiomètre

radiometric quantity

grandeur énergétique

radiometric resolution - Syn. de gray-level resolution.

radiometry

radiométrie

random noise - Syn. de random walk noise.

random picture noise - Syn. de random walk noise.

random variable

variable aléatoire

random walk noise, random noise,

bruit aléatoire

random picture noise

range - Syn. de depth dimension.

range finding, range sensing

télémétrie

range image, depth map, range map,

depth image

image de profondeur, carte de profondeur, carte de profondeurs

range map - Syn. de range image.

range of accomodation

latitude de mise au point, intervalle d'accommodation

range sensing - Syn. de range finding.

range-finder, ranging system,

ranging device

télémètre

ranging device - Syn. de range-finder.

ranging system - Syn. de range-finder.

ranked alphabet

alphabet à degrés

raw image

ray optics, geometrical optics

image brute

optique géométrique

reading glass, hand glass

loupe de lecture 
receptor cell, photoreceptor,

récepteur physiologique

light receptor

reciprocal wavelength - Syn. de wave number.

recognition grammar

grammaire de reconnaissance

reconstruction from projections

reconstruction d'image à partir de ses projections

rectangle function

signal créneau, fonction porte,

signal rectangulaire, signal porte, créneau

rectangular lattice,

rectangular sampling lattice

réseau rectangulaire,

réseau d'échantillonnage rectangulaire

rectangular sampling lattice - Syn. de rectangular lattice.

red shift

décalage vers le rouge

reduced eye

œil réduit

reference vector - Syn. de prototype.

reference wave

reflectance, reflection factor, scene radiance, radiant reflectance

reflecting telescope, telescope,

reflector

reflection factor - Syn. de reflectance.

reflector - Syn. de reflecting telescope.

refracting telescope,

refracting terrestrial telescope,

refractor telescope

refractive index, index of refraction

refrangibility, Abbe number

onde de référence

réflectance, réflectance de surface, pouvoir de réflectance

télescope, télescope à réflexion

region - Syn. de image region.

region boundary, boundary line, region outline

region growing

region merging,

merge-only quad-tree segmentation

lunette, lunette terrestre,

lunette d'approche

indice de réfraction, indice absolu

réfrangibilité

contour, ligne de contour, contour d'une région

segmentation par reconstitution des régions

méthode par fusion

region outline - Syn. de region boundary.

region segmentation,

region-based segmentation

segmentation d'images en régions, segmentation d'image en régions, approche par les régions, segmentation basée sur les régions

region splitting, split-only quad-tree, segmentation segmentation par division, division d'images 
region-based segmentation - Syn. de region segmentation.

regular grammar, type 3 grammar, finite-state grammar grammaire régulière, grammaire de Kleene, grammaire rationnelle, grammaire de type 3, grammaire d'états finis

regular reflection - Syn. de specular reflection.

relaxation

méthode de relaxation

relaxation algorithm

algorithme de relaxation

remote sensing

télédétection

representation

représentation

representation pattern vector - Syn. de pattern vector.

resampling

rééchantillonnage

resolution - Syn. de resolving power.

resolution

résolution

resolving power - Syn. de power of resolution.

resolving power, resolution

résolution d'un film

retinal image

image rétinienne

reverse Fourier - Syn. de inverse Fourier transform.

reverse Fourier transform - Syn. de inverse Fourier transform.

reversibility of light paths

loi du retour inverse de la lumière

revolving nosepiece

tourelle porte-objectifs, monture à revolver

rewriting rule - Syn. de production.

right angle prism

prisme à angle droit

right-hand, dextrorotary

dextrogyre

rod

bâtonnet

root - Syn. de root node.

root node, start symbol,

racine, ancêtre, tête

root symbol, top node, root

root symbol - Syn. de root node.

row vector

vecteur-ligne

rubber mask - Syn. de flexible template.

rubber sheet transformation - Syn. de rubber-sheet deformation.

rubber-sheet deformation, déformation du plan rubber-sheet distortion, rubber sheet transformation, rubber-sheet transformation, rubber-sheet distorsion

rubber-sheet distorsion - Syn. de rubber-sheet deformation. 
rubber-sheet distortion - Syn. de rubber-sheet deformation.

rubber-sheet transformation - Syn. de rubber-sheet deformation.

ruby laser

laser à rubis, laser à cristaux de type rubis

ruled surface

surface réglée

run length coding, run-length coding

codage par plage

run-length coding - Syn. de run length coding.

running wave, traveling wave

onde progressive

S I N ratio - Syn. de signal-to-noise ratio.

sample pattern, learning sample,

training sample, training sample pattern

sample point - Syn. de point sampling.

sample set - Syn. de training set.

sampler

sampling

sampling aperture

sampling function

sampling grid - Syn. de sampling lattice.

sampling interval

sampling lattice, spatial sampling grid, de spatial sampling lattice,

sampling grid

sampling rate

sampling theorem,

Shannon sampling theorem,

Nyquist criterion

SAR - Syn. de synthetic aperture radar.

satellite imagery

saturation

scalar product - Syn. de inner product.

scaling, image scaling

scanning

scanning device

scanning electron microscope, SEM

scattering échantillon de référence, échantillon d'apprentissage

échantillonneur
échantillonnage

ouverture de balayage,

ouverture du faisceau de balayage

fonction d'échantillonnage

pas d'échantillonnage,

période d'échantillonnage, pas de discrétisation

réseau d'échantillonnage, maillage de discrétisation, réseau de discrétisation

fréquence d'échantillonnage

théorème de l'échantillonnage,

théorème de l'interpolation de Shannon

imagerie spatiale, imagerie par satellite, imagerie satellitaire

saturation

changement d'échelle

balayage, balayage d'image

système de balayage

microscope électronique à balayage

diffusion 
scene analysis,

automatic scene analysis,

image understanding

scene brightness - Syn. de radiance.

scene irradiance - Syn. de irradiance.

scene radiance - Syn. de reflectance.

scotopic vision, dark-adapted eye vision

$S D F$ - Syn. de slope density function.

search space

search tree

segment - Syn. de image region.

segmentation

segmentation technique

segmented image

Seidel aberration

seismic pattern recognition analyse de scène

vision nocturne, vision scotopique

espace de recherche

arbre de recherche

segmentation

technique de segmentation

image segmentée

aberration de Seidel

reconnaissance de signaux sismiques

SEM - Syn. de scanning electron microscope.

semantic knowledge

connaissance sémantique

semi-simple path - Syn. de trail.

sensing device - Syn. de sensor.

sensitivity center - Syn. de development center.

sensitizer

sensibilisateur

sensor, sensing device

capteur

sensor resolution

résolution (d'un capteur)

sensor response

réponse d'un capteur

sensor sensitivity

sensibilité d'un détecteur

sensor spectral intensity response,

réponse spectrale

spectral response

phrase, liste, chaîne, mot

sentence, word, string

développement en série

set

ensemble

set operation, Boolean set operation

opération ensembliste

set theory

théorie des ensembles, théorie ensembliste

set union, union

réunion, union

shadow

ombre

Shannon sampling theorem - Syn. de sampling theorem. 
shape

shape number

shaping-from-shading method

sharp edge

sharpening, deblurring

sharpness, image sharpness

shift invariant, isoplanatic, space invariant

shift register

shot noise

shrinking - Syn. de erosion.

signal

signal processing

signal theory

signal's frequency spectrum

signal-to-noise ratio, image $S N R$,

SNR, S/N ratio

signature

significant feature silhouette, silhouette image

silhouette image - Syn. de silhouette.

silver halide

similarity measure,

pattern similarity measure forme d'objet, forme

nombre de forme

inférence de formes à partir d'ombres

arête vive

renforcement des contours, accentuation des contours

netteté d'une image

invariant par translation

registre à décalage

bruit de gramulation

signal

traitement du signal

théorie du signal,

théorie du traitement du signal

spectre de fréquences, spectre de Fourier

rapport signal/bruit

signature

caractéristique pertinente

simple graph - Syn. de undirected graph.

simple path

chemin simple

sine condition - Syn. de isoplanetic condition.

skeleton

squelette

skeleton operation - Syn. de skeletonization.

skeletonization,

squelettisation

medial axis transformation,

halogénure d'argent, halosel d'argent

indice de ressemblance,

mesure de similarité, indice de similitude

skeleton operation,

medial axis transform, MAT

slope density function, SDF

fonction de pente 
smoothed image

smoothing, image smoothing

smoothness constraint

snapshot

Snell's law

SNR - Syn. de signal-to-noise ratio.

Sobel edge detector - Syn. de Sobel operator.

Sobel operator, Sobel edge detector

Sobel template, gradient Sobel template

solid angle

solid-state camera

solid-state sensor

space invariant - Syn. de shift invariant.

space-invariance

spatial coherence image lissée

lissage

contrainte physique de continuité, contrainte de continuité

image instantanée

loi de Descartes

spatial convolution - Syn. de group processing.

spatial differentiation

opérateur de Sobel

matrice de Sobel

angle solide

caméra à état solide, caméra à semi-conducteurs, caméra à l'état solide,

caméra à semiconducteurs, caméra solide

capteur solide, capteur à semi-conducteurs, capteur à semiconducteurs

spatial domain

différenciation spatiale

spatial filter

domaine spatial

spatial filtering

filtre spatial

invariance par translation, stationnarité

cohérence spatiale

spatial frequency.

two-dimensional frequency

spatial frequency filtering, spatial filtering

spatial local operation, group processing operation

spatial occupancy array

spatial resolution

spatial sampling, image tesselation, tesselation discrétisation

filtering.

fréquence spatiale

filtrage spatial

opération locale

tableau d'occupation spatiale

résolution spatiale

échantillonnage spatial, spatiale

spatial sampling grid - Syn. de sampling lattice.

spatial sampling lattice — Syn. de sampling lattice.

spatial spectrum - Syn. de spectrum. 
spectral band - Syn. de frequency band.

spectral distribution

spectral filter

spectral line

spectral luminous efficiency composition spectral, répartition spectrale

filtre spectral

raie

efficacité lumineuse spectrale

spectral response - Syn. de sensor spectral intensity response.

spectroscopic parallax

parallaxe spectroscopique

spectrum, image spectrum,

image spatial spectrum, spatial spectrum

specular density

spectre (d'une image)

specular reflection, regular reflection, direct reflection, mirror reflexion

specular surface, glossy surface

densité spéculaire

réflexion métallique

surface métallique

speed - Syn. de sensitivity.

speed

vitesse, sensibilité

spherical wave

onde sphérique

split-only quad-tree segmentation - Syn. de region splitting.

spot of light, light spot

spot

$S P R$ - Syn. de statistical pattern recognition.

spread function - Syn. de impulse response.

squaring

stadimeter

stage

stand

standard deviation

standing waves

start symbol - Syn. de root node.

state space, problem space, domain

state transition diagram

state transition matrix

state-conditional probability

density function

stationary random process

statistic

statistical classification élévation au carré

lunette stadimétrique, stadimètre

platine, porte-objet

statif

écart-type

ondes stationnaires

espace d'états

diagramme de transition

matrice de transition

fonction de densité conditionnelle

processus aléatoire stationnaire

paramètre statistique, caractéristique statistique

classification statistique, discrimination statistique 
statistical pattern recognition, $S P R$

Stefan-Boltzmann law

stepped lenses - Syn. de Fresnel lens.

stereo disparity, binocular disparity,

disparity

stereo fusion - Syn. de stereoscopic vision.

stereo image

stereo matching

stereo pair

stereo vision - Syn. de stereoscopic vision.

stereogram

stereographic projection

stereopsis - Syn. de stereoscopic vision.

stereoscope

stereoscopic vision, stereo fusion,

stereo vision, stereovision,

stereopsis vision

stereovision - Syn. de stereoscopic vision.

still camera

stochastic grammar

stochastic process

stop, lens stop

stop down (to)

straight line segment - Syn. de line segment.

straight-line travel of light

string - Syn. de sentence.

string

string representation

stroke

structural description, image structural description

structural pattern recognition - Syn. de syntactic pattern recognition.

structural property - Syn. de structural feature. propagation rectiligne de la lumière

chaîne

reconnaissance par classification statistique

loi de Stefan-Boltzmann

disparité

image stéréo, image stéréoscopique stéréocorrespondance, appariement stéréoscopique, mise en correspondance stéréoscopique, mise en correspondance d'images stéréoscopiques, mise en correspondance d'une paire d'images stéréo

paire d'images stéréo, couple stéréoscopique

stéréogramme

projection stéréographique

stéréoscope

stéréovision, stéréo, stéréoscopie, vision stéréoscopique

appareil photographique

grammaire stochastique

processus stochastique

diaphragme

diaphragmer

description en chaîne

trait

description structurelle 


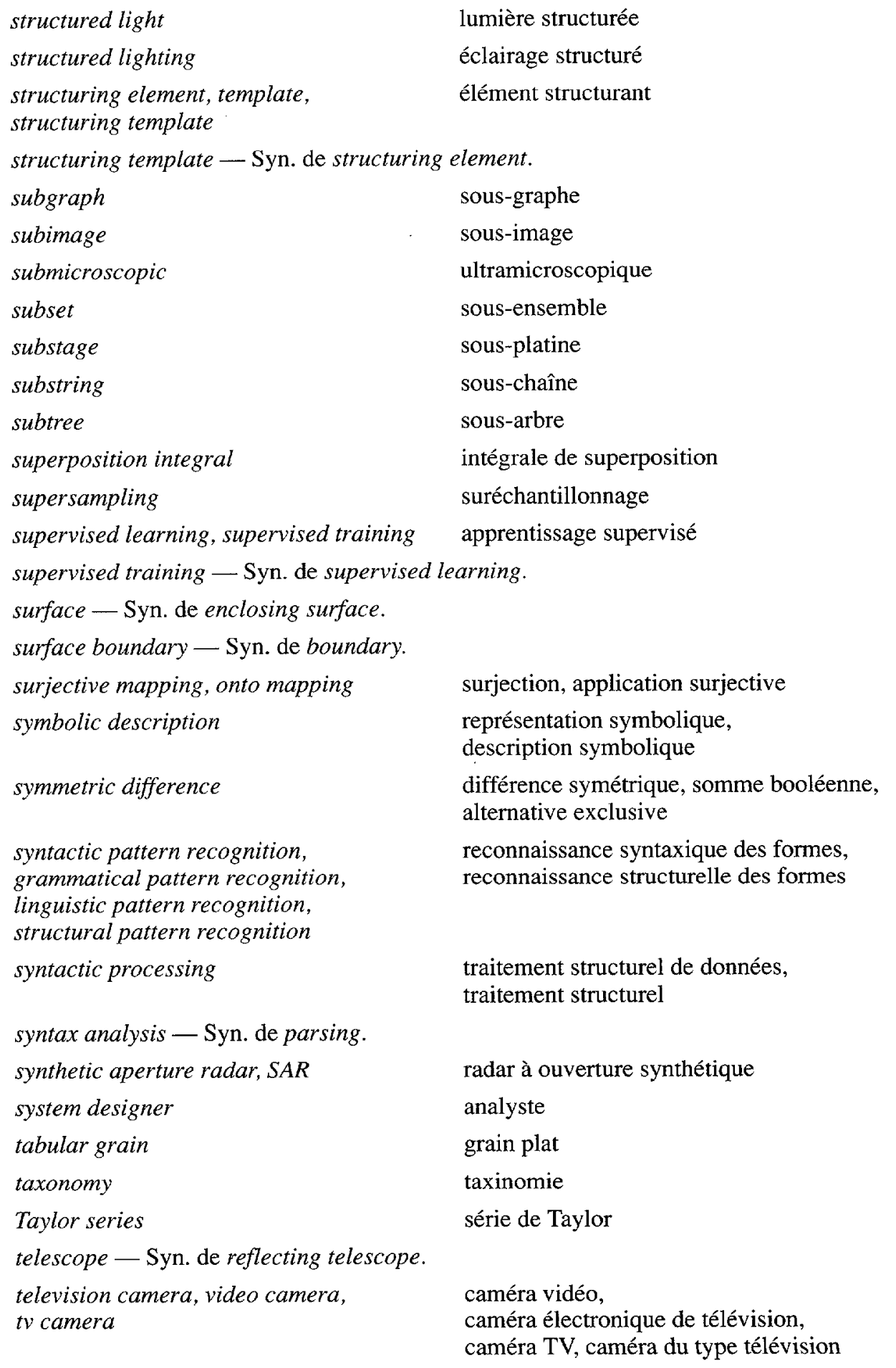


television image - Syn. de TV image.

television picture - Syn. de TV image.

template - Syn. de structuring element.

template, window, mask, prototype, filtre, fenêtre, masque

matching, template, filter

template filtering - Syn. de matched filtering.

template matching - Syn. de matched filtering.

templating - Syn. de matched filtering.

terminal - Syn. de terminal symbol.

terminal node, tip node, leaf

feuille, sommet pendant, nœud terminal

terminal sentence, terminal string

chaîne terminale

terminal set - Syn. de terminal vocabulary.

terminal shape element - Syn. de primitive shape.

terminal string — Syn. de terminal sentence.

terminal symbol, constant, terminal

symbole terminal

lerminal vocabulary, terminal set

vocabulaire terminal, alphabet terminal

lesselation - Syn. de spatial sampling.

texture

texture

texture gradient

gradient de texture

texture primitive

primitive de texture, textel

thickening

épaississement

thinning

three-dimensional object

amincissement

three-dimensional perception

objet tridimensionnel

perception tridimensionnelle

three-dimensional reconstruction,

$3 D$ reconstruction

threshold, threshold level

reconstruction d'images tridimensionnelles, reconstruction tridimensionnelle

threshold level - Syn. de threshold.

thresholded gradient image

seuil, seuil de binarisation

thresholding

image du gradient seuillé

time integral

seuillage

time of flight

intégrale par rapport au temps

temps de vol, temps d'aller-retour

time-varying imagery — Syn. de dynamic imagery.

token

symbole

tomogram

tomogramme

tomography

tomographie 
toolmaker's microscope

microscope d'atelier

top node - Syn. de root node.

topological descriptor - Syn. de topological property.

topological property,

topological descriptor

propriété topologique,

paramètre topologique,

descripteur topologique,

caractéristique topologique

topological space

espace topologique

total curvature - Syn. de Gaussian curvature.

total internal reflection

réflexion totale

tournament

tournoi

trail, semi-simple path, path, chain

chaîne (dans un graphe)

training pattern set - Syn. de training set.

training phase, classifier design,

phase d'apprentissage

learning phase

training process - Syn. de learning.

training sample - Syn. de sample pattern.

training sample pattern - Syn. de sample pattern.

training set, sample set,

ensemble d'apprentissage

training pattern set

transfer function

fonction de transfert, gain complexe

transit

théodolite

transitive closure

fermeture transitive

translucent

translucide

transmission cafficient - Syn. de transmittance.

transmission electron microscope,

microscope électronique à transmission

conventional electron microscope

transmission factor - Syn. de transmittance.

transmittance, transmission ccefficient,

transmission factor

transmittance, facteur de transmission, transmission optique, transparence

transparency

diapositif

transverse wave

onde transversale

trapping site - Syn. de development center.

traveling wave - Syn. de running wave.

tree automaton

automate d'arbre

tree grammar

grammaire d'arbre

tree grammar inference

inférence de grammaires d'arbres 
tree graph - Syn. de tree.

tree search

tree structure

tree to tree distance

triangular sampling grid

triangulation

trinocular vision

Turing machine

tv camera - Syn. de television camera.

TV image, television picture, television image

twin

two point perspective, angular perspective

two-dimensional comb, Dirac comb parcours d'un arbre, exploration d'arbre, recherche arborescente

structure d'arbre

distance entre arbres

réseau d'échantillonnage triangulaire

triangulation

stéréovision trinoculaire

machine de Türing

image de télévision

macle

perspective oblique

signal peigne de Dirac

two-dimensional frequency - Syn. de spatial frequency.

two-dimensional picture

image en $2 \mathrm{D}$, image numérique $2 \mathrm{D}$, image bidimensionnelle

two-dimensional sensor

capteur 2D

two-humped grey-level histogram - Syn. de bimodal histogram.

two-humped histogram - Syn. de bimodal histogram.

type 0 grammar - Syn. de unrestricted grammar.

type 1 grammar - Syn. de context-sensitive grammar.

type 2 grammar - Syn. de context-free grammar.

type 3 grammar - Syn. de regular grammar.

ultramicroscope

ultramicroscope

ultrasonic ranging, ultrasound ranging

télémètre laser

ultrasound ranging - Syn. de ultrasonic ranging.

ultraviolet

ultraviolet proche

unaided viewing,

observation à l'œil nu

viewing without optical aid,

naked eye viewing

underexposure

sous-exposition

undersampling

sous-échantillonnage

undirected graph, simple graph

graphe non orienté, graphe simple

union - Syn. de set union. 
uniqueness constraint

unrestricted grammar, type 0 grammar contrainte d'unicité

grammaire sans restriction, grammaire de type 0

unsupervised classification - Syn. de unsupervised learning.

unsupervised classification clustering

unsupervised learning, clustering, unsupervised classification

vacuum ultraviolet, extreme ultraviolet

valency - Syn. de degree.

vanishing line

variance - Syn. de cluster variance.

vector - Syn. de pattern vector.

vector head

vector product - Syn. de cross product.

vector space

vector tail

vertex - Syn. de node.

vertex

video camera - Syn. de television camera.

vidicon - Syn. de vidicon camera.

vidicon camera, vidicon

vidicon tube

viewer, observer

viewer

viewing screen

vignetting classification non supervisée

apprentissage non supervisé

ultraviolet lointain

ligne de fuite

origine d'un vecteur

espace vectoriel

extrémité d'un vecteur

sommet

visibility efficiency - Syn. de luminosity efficiency.

visible image

image physique visible

visible light

visible spectrum

lumière visible

visual acuity

spectre visible

visual cue

acuité visuelle

indice visuel

visual data - Syn. de visual information.

visual image

visual information, visual data

image visuelle

information visuelle, donnée visuelle

visual instrument 


$\begin{array}{ll}\text { visual perception } & \text { perception visuelle } \\ \text { visual sensation } & \text { impression visuelle }\end{array}$

visual system - Syn. de computer vision system.

volume element - Syn. de voxel.

voxel, volume element

voxel

Walsh-Hadamard transform

transformée de Walsh-Hadamard, TWH

watch maker's glass

loupe d'horloger

wave front - Syn. de wave surface.

wave number, reciprocal wavelength nombre d'onde

wave surface, wave front surface d'onde

wave train

train d'ondes

wavefront-reconstruction imaging - Syn. de holography.

wavelength

longueur d'onde

wavelet

ondelette de Huyghens

weakly convex hull

enveloppe faiblement convexe

weakly-connected graph - Syn. de connected graph.

wheel

roue

white light

lumière blanche

white noise

bruit blanc,

bruit à corrélation microscopique

whitening

blanchiment

wide-angle lens - - Syn. de wide angle.

wide-angle lens

objectif grand angulaire, grand angulaire, grand angle

Wiener spectrum - Syn. de power spectrum.

window - Syn. de template.

word - Syn. de sentence.

work function

travail d'extraction

X-ray imaging

imagerie rayons $\mathrm{X}$

zero crossing, zero-crossing

passage par zéro

zero-crossing - Syn. de zero crossing.

JACQues LethuILlier et MONIQUe C. CORMIER

Université de Montréal, Montréal, Canada

\section{RÉFÉRENCES}

ALEKSANDER, Igor (1984) : Artificial Vision for Robots, New York, Chapman and Hall, $233 \mathrm{p}$.

ANDREWS, Harry C. (1972): Mathematical Techniques in Pattern Recognition, New York, Wiley, 242 p.

ANZAI, Yuichiro (1989) : Pattern Recognition and Machine Learning, New York, Academic Press, 407 p. 
ARQUES, P.-Y. (1982): Décisions en traitement du signal, Paris, Masson, $288 \mathrm{p}$

AYACHE, Nicholas (1989): Vision stéréoscopique et perception multisensorielle, Paris, InterEditions, 345 p.

BALLARD, Dana H. et Christopher M. BROWN (1982): Computer Vision, Englewood Cliffs, Prentice-Hall, $523 \mathrm{p}$.

BAXES, Gregory A. (1984): Digital Image Processing: a Practical Primer, Englewood Cliffs, Prentice-Hall, $182 \mathrm{p}$.

BELAID, Abdel et Yolande BELAID (1992) : Reconnaissance des formes, Paris, Interéditions, $429 \mathrm{p}$.

BENVENISTE, Albert et Michel MÉTIVIER (1987): Algorithmes adaptatifs et approximations stochastiques à l'identification, au traitement du signal et à la reconnaissance des formes, Paris, Masson, $367 \mathrm{p}$.

BENZÉCRI, J.-P. (1973): L' analyse des données - l - la taxinomie, Paris, Dunod, $615 \mathrm{p}$.

BENZÉCRI, J.-P. (1973): L'analyse des données - 2 - L'analyse des correspondances, Paris, Dunod, 619 p.

BERTIER, P. et J.-M. BOUROCHE (1975) : Analyse des données multidimensionnelles, Paris, PUF, 270 p.

BESANCON, Jacques E. (1988) : Vision par ordinateur en deux et trois dimensions, Paris, Eyrolles, 410 p.

BETTON, Gérard (1985) : La photomicrographie, Paris, PUF, 127 p.

BETTON, Gérard (1975): La photographie scientifique, Paris, PUF, $128 \mathrm{p}$

BOLC, Leonard et Zenon KULPA (1981) : Digital Image Processing Systems, Berlin, Springer-Verlag, 353 p.

BOYLE, Roger D. et Richard C. THOMAS (1988): Computer Vision: A First Course, Oxford, Blackwell Scientific, $210 \mathrm{p}$.

BROWN, Christopher (1988): Advances in Computer Vision - Volume 1, Hillsdale, Lawrence Erlbaum Associates, $233 \mathrm{p}$.

BROWN, Christopher (1988): Advances in Computer Vision - Volume 2, Hillsdale, Lawrence Erlbaum Associates, $186 \mathrm{p}$.

BUNDY, Alan (1986) : Catalogue of Artificial Intelligence Techniques, Berlin, Springer-verlag, $182 \mathrm{p}$

CLAVAUD, Richard et Jean-Paul GILLET (1984) : Images: Visualisation et Informatique, Paris, Centre d'études des systèmes et des technologies avancées, $76 \mathrm{p}$.

COHEN, Paul (1988) : Analyse d'images et vision par ordinateur, Montréal, École Polytechnique de Montréal, $57 \mathrm{p}$.

COIFFET, Philippe (1981) : Les robots - tome 2 : Interaction avec l'environnement, Paris, Hermès, $283 \mathrm{p}$.

COSTER, Michel et J. L. CHERMANT (1989): Précis d'analyse d' images, Paris, Presses du C.N.R.S., 560 p.

COUZY, Alain (1981): La télédétection, Paris, PUF, 128 p.

DAVIES, E. Roy (1990) : Machine Vision: Theory, Algorithms, Practicalities, London, Academic Press, 547 p.

DESVIGNES, F. (1987) : Détection et détecteurs de rayonnements optiques, Paris, Masson, 346 p.

DESVIGNES, François (1991) : Rayonnements optiques: radiométrie, photométrie, Paris, Masson, 311 p.

DIDAY, E. et Y. LECHEVALIER (1978): Classification automatique et perception par ordinateur, Rocquencourt, Institut de recherche d'informatique et d'automatique, $111 \mathrm{p}$.

DOUGHERTY, Edward R. et Charles R. GIARDINA (1987): Matrix Structured Image Processing, Englewood Cliffs, Prentice-Hall, $258 \mathrm{p}$.

DUFFIEUX, P. M. (1970) : L'intégrale de Fourier et ses applications à l'optique, Paris, Masson, 172 p.

EKSTROM, Michael P. (1984) : Digital Image Processing Techniques, Orlando, Academic Press, 372 p.

EVERITT, Brian (1974) : Cluster Analysis, New York, Halsted Press (Wiley), $136 \mathrm{p}$.

FAURE et al. (1985): Perception et reconnaissance des formes, Paris, Editest, $286 \mathrm{p}$.

FOLEY, James et al. (1990): Computer Graphics - Principles and Practice, Reading, Addison-Wesley, 1174 p.

FRANÇON, Maurice (1970): Vibrations lumineuses - Optique cohérente, Paris, Masson, $260 \mathrm{p}$.

FREEMAN, Herbert (1990): Machine Vision for Three-Dimensional Scenes, Boston, Academic Press, $419 \mathrm{p}$.

FU, K. S. et A. B. WHINSTON (1977) : Pattern Recognition Theory and Application, Leyden, Noordhoff International Publishing,

FU, K. S. (1980) : Digital Pattern Recognition, Berlin, Springer-Verlag, 236 p.

FU, King Sun (1982): Syntactic Pattern Recognition and Applications, Englewood Cliffs, Prentice-Hall, 596 p. FUKUNAGA, Keinosuke (1990): Introduction to Statistical Pattern Recognition, Boston, Academic Press, 591 p.

GASPART, P. (1986): Vision et intelligence artificielle: clefs de l'adaptivité en productique, Université de Liège, $276 \mathrm{p}$.

GENTHON, Ph. (1989) : Dictionnaire de l' IA, Paris, Hermès, 156 p.

GONZALEZ, Rafael C. et Michael G. THOMASON (1978) : Syntactic Pattern Recognition-An Introduction, Reading, Addison-Wesley, $283 \mathrm{p}$.

GOODMAN, J. W. (1968) : Introduction to Fourier Optics, New York, McGraw-Hill, 287 p.

GRAY, H. J. et Alan ISAACS (1975) : A New Dictionary of Physics, London, Longman Group Limited, 619 p.

HALL, Ernest L. (1979): Computer Image Processing and Recognition, New York, Academic Press, 584 p.

HARALICK, Robert M. et SHAPIRO, Linda G. (1992): Computer and Robot Vision, Reading, AddisonWesley, $672 \mathrm{p}$.

HARARY, Frank (1969) : Graph Theory, Reading, Addison-Wesley, 273 p.

HORN, Berthold Klaus Paul (1986) : Robot Vision, Cambridge, The MIT Press, 509 p. 
HUANG, Thomas S. (1986) : Image Enhancement and Restoration, Greenwich, JAI Press, 378 p.

HUANG, Thomas S. (1984): Image Reconstruction from Incomplete Observations, Greenwich, JAI Press, $344 \mathrm{p}$.

JAMBU, M. (1978): Classification automatique pour l'analyse des données - 1. Méthodes et algorithmes, Paris, Dunod, 312 p.

JAMES, Mike (1988) : Pattern Recognition, New York, Wiley, 144 p.

JAMES, Mike (1985) : Classification Algorithms, New York, Wiley, 211 p.

KANAL, Laveen N. et Azriel ROSENFELD (1981): Progress in Pattern Recognition, Amsterdam, NorthHolland,

KASTURI, Rangachar et Ramesh C. JAIN (1991) : Computer Vision: Principles, Washington, IEEE Computer Society Press, $711 \mathrm{p}$.

KODRATOFF, Yves et Michel BARES (1991) : Base terminologique de l'intelligence artificielle, Paris, Technique et documentation - Lavoisier, $122 \mathrm{p}$.

KRISHNAIAH, P. R. et L. N. KANAL (1982) : Handbook of Statistics 2 - Classification Pattern Recognition and Reduction of Dimensionality, Amsterdam, North-Holland, 903 p.

LABELLE, Jacques (1983): Théorie des graphes, Montréal, Modulo Éditeur, $183 \mathrm{p}$.

LACOUME, Jean-Louis (1983): Théorie du signal, Paris, PUF, $128 \mathrm{p}$.

LANNES, A. et J. Ph. PÉREZ (1983) : Optique de Fourier en microscopie électronique, Paris, Masson, $216 \mathrm{p}$.

LAURGEAU, Claude et Michel PARENT (1985): Les machines de vision en robotique, Strasbourg, Éditions techniques d'Alsace, $281 \mathrm{p}$.

LERESCHE, Georges (1972): Initiation à la théorie des graphes, Genève, Librairie Droz, 73 p.

LEVINE, Martin D. (1985): Vision in Man and Machine, New York, McGraw-Hill, 574 p.

LINDLEY, Craig A. (1991): Practical Image Processing in C, New York, Wiley, 554 p.

LOW, Adrian (1991): Introductory Computer Vision and Image Processing, London, McGraw-Hill, $244 \mathrm{p}$.

MARION, André (1987) : Introduction aux techniques de traitement d'images, Paris, Eyrolles, 279 p.

MARR, David (1982) : Vision: A computational Investigation into the Human Representation and Processing of visual Information, San Francisco, Freeman, 397 p.

MARÉCHAL, André (1952): Traité d'optique instrumentale - Imagerie géométrique et aberrations, Paris, Éditions de la Revue d'Optique théorique et instrumentale, $244 \mathrm{p}$.

MEISEL, William S. (1972): Computer-Oriented Approaches to Pattern Recognition, New York, Academic Press, $250 \mathrm{p}$.

MENDEL, J. M. et FU, K. S. (1970) : Adaptive, Learning, and Pattern Recognition Systems: Theory and Applications, New York, Academic Press, $446 \mathrm{p}$.

MERCADAL, Dennis (1990): Dictinary of Artificial Intelligence, New York, Van Nostrand Reinhold, 334 p.

MICLET, L. (1984): Méthodes structurelles pour la reconnaissance des formes, Paris, Eyrolles, $184 \mathrm{p}$.

MÉZIN, Gérard (1988) : La vision par ordinateur dans l'industrie, Paris, Hermès, 64 p.

NIEMANN, Heinrich (1990): Pattern Analysis and Understanding, New York, Springer-Verlag, 371 p.

PAJANI, Dominique (1989): Mesure par thermographie infrarouge, Chatenay Malabry 92290, Add Éditeur, $418 \mathrm{p}$.

PAU, L. F. et M. Y. EL NAHAS (1983): An Introduction to Infrared Image Acquisition and Classification Systems, New York, Wiley, $268 \mathrm{p}$.

PAVEL, Monique (1989) : Fundamentals of Pattern Recognition, New York, M. Dekker, 183 p.

PAVLIDIS, Theo (1982) : Algorithms for Graphics and Image Processing, Rockville, Computer Science Press, $416 \mathrm{p}$.

PELAT, Alain (1988): Imagerie et télévision - Une introduction: vision, photométrie, colorimétrie, Paris, Édition marketing, $175 \mathrm{p}$.

POSTAIRE, Jack-Gérard (1987) : De l'image à la décision, Paris, Dunod, $190 \mathrm{p}$

PRATT, William (1991): Digital Image Processing, New York, Wiley, 698 p.

PUGH, Alan (1983): Robot Vision, Berlin, Springer-Verlag, 356 p.

ROSENFELD, Azriel (1984): Multiresolution Image Processing and Analysis, Berlin, Springer-Verlag, 385 p.

ROSENFELD, Azriel et Avinash C. KAK (1982): Digital Picture Processing, New York, Academic Press, 436 p.

ROSENFELD, Azriel et Avinash C. KAK (1982): Digital Picture Processing, New York, Academic Press, $350 \mathrm{p}$.

SANDERSON, Ronald J. (1983): Machine Vision Systems: a Summary and Forecast, Naperville, Tech Tran Corp, $162 \mathrm{p}$.

SCHALKOFF, Robert J. (1989) : Digital Image Processing and Computer Vision, New York, Wiley, 489 p.

SCHALKOFF, Robert (1992): Pattern Recognition - Statistical, Structural and Neural Approaches, New York, Wiley, $364 \mathrm{p}$.

SEGURA, Jean (1989): Du scanner aux images numériques, Paris, Nathan Communication, 111 p.

SERRA, J. (1969): Introduction à la morphologie mathématique, Paris, École Nationale Supérieure des Mines de Paris, $160 p$.

SHIRAI, Yoshiaki (1987) : Three-dimensional Computer Vision, Berlin, Springer-Verlag, $297 \mathrm{p}$.

SIMON, J.-C. (1984) : Reconnaissance des formes par algorithmes, Paris, Masson, $251 \mathrm{p}$. 
TERRIEN, Jean (1962): Les mesures physiques, Paris, PUF, $128 \mathrm{p}$

TERRIEN, Jean et François DESVIGNES (1972): La photométrie, Paris, PUF, $128 \mathrm{p}$.

THERRIEN, Charles W. (1989) : Decision Estimation and Classification, New York, Wiley, 251 p.

THOMAS, Ian L. et al. (1987) : Classification of Remote Sensed Images, Bristol, A. Hilger, 268 p.

TOU, J. T. et R. C. GONZALEZ (1974) : Pattern Recognition Principles, Reading, Addison-Wesley,

TOUMAZET, Jean-Jacques (1988) : Traitement de l' image sur micro-ordinateur, Paris, Sybex, $260 \mathrm{p}$.

UHR, Lconard (1987) : Parallel Computer Vision, Boston, Academic Press, 303 p.

VOGT, Robert C. (1989) : Automatic Generation of Set Recognition Algorithms, New York, Springer-Verlag, $275 \mathrm{p}$.

WATT, Roger J. (1991) : Pattern Recognition by Man and Machine, London, Macmillan, 147 p.

WECHSLER, Harry (1990) : Computational Vision, Boston, Academic Press, 558 p.

WEGMAN, Edward J. et Douglas J. DE PRIEST (1986): Statistical Image Processing and Graphics, New York, M. Dekker, 351 p.

WILSON, Robin J. (1979): Introduction to Graph Theory, New York, Academic Press, $163 \mathrm{p}$

WOODS, Richard E. et Rafael C. GONZALEZ (1992): Digital Image Processing, Reading, Addison-Wesley, $716 \mathrm{p}$.

ZUECH, N. et R. MILLER (1987) : Machine Vision, Lilburn (Georgia), The Fairmount Press, 209 p. 